\title{
RETOS FILOSÓFICOS EN EL HORIZONTE POSTMODERNO
}

José Luis Rodríguez García

Universidad de Zaragoza

\section{La crisis finisecular}

Las alteraciones sociales y culturales no se manifiestan como catástrofes, sino como sutil ebriedad que se revela como potencia cuando los ecos de lo viejo ceden su privilegio a las exigencias de lo que comienza a aparecer. Siempre son numerosos los síntomas que anuncian el murmullo en el subsuelo y, en consecuencia, podría analizarse toda crisis a partir de la lectura de cualquier síntoma. Pues bien, las postreras décadas del siglo XX dejaron constancia de una inquietud notable. ¿De qué nos hablaban los síntomas? Tomemos uno al azar -como suele afirmarse retóricamente-. Rememora Badiou en su Manifiesto por la filosofía que «la poesía ha cargado con ciertas funciones de la filosofía»" Nada parece más cierto, si subrayamos la generosa atribución heideggeriana del decir fundamental a lo poético o recordamos la radical y asentada convicción de Deleuze para quien son Hölderlin, Kleist, Michaux, Kafka, Artaud o Lawrence quienes escribieron «le roman du spinozisme»². Pero contra el sentido vicario de la poesía se expresaba el propio Badiou: «yo mantengo que la edad de los poetas concluyó»3. Tal síntoma nos habla: por un lado, la filosofía se oculta para abolir cualquier pleito competencial con la poesía, pero, por otra parte, la filosofía reivindica un añorado lugar en la liturgia de los saberes.

Pensar que reivindicar el reinado de los poetas o proceder a la reubicación de la aventura filosófica es una polémica entre filósofos y poetas sería banal.

\footnotetext{
${ }^{1}$ BADIOU A., Manifiesto por la filosofía, Madrid, Tecnos, 1990, p. 49.

${ }^{2}$ DELEUZE G. - GUATTARI F., Qu'est-ce que la philosophie?, Paris, Minuit, 1991, p. 65.

${ }^{3}$ BADIOU A., Manifiesto por la filosofía, p. 51.
} 
Pues un asunto más esencial revela el síntoma ¿De qué se trata? El propio Badiou lo ha subrayado de manera rotunda cuando advertía que «es necesario invertir el diagnóstico nietzscheano. El siglo y Europa deben imperativamente sanar del antiplatonismo ${ }^{4}$. La alusión a la enfermedad del antiplatonismo no puede pasar desapercibida. Es clave ya que, en efecto, la filosofía se rebeló contra la arquitectura platónica y sus innúmeras variantes a lo largo de las últimas décadas del siglo XX. De Adorno a Deleuze... Las páginas más encendidas de Dialéctica negativa recomiendan asumir el imperativo teórico contra la estrategia platónica, ya que «la situación histórica hace que la filosofía tenga su verdadero interés allí precisamente donde Hegel, de acuerdo con la tradición, proclamó su indiferencia en lo carente de concepto, en lo particular y especial, eso que desde Platón fue despachado como perecedero y sin importancia, para serle colgada al fin por Hegel la etiqueta de existencia corrompida $»^{5}$. Estrategia antiplatónica que Deleuze reiterará con alegre suficiencia al advertir que «toda reacción contra el platonismo es un restablecimiento de la inmanencia en su extensión y en su pureza, que prohíbe el retorno de un transcendente $»^{6}$.

Ah, ¿resulta entonces que se oculta otra polémica tras la renovada atmósfera fiscalizadora contra el orgullo de lo poético? Insistamos un momento en la consideración de lo que hemos venido a presentar como advertencia de una catástrofe. No asistimos a la renovación de una disputa escolástica, si bien es cierto que la literatura platónica resulta ser el infinito de la escritura -pues aún no sabemos escribir más allá de Platón: acaso es que no podemos escribir más allá de Platón-. Sucede que el debate platónico se ha revelado como problemática política. Cuando Deleuze afrontó las motivaciones últimas de la opción platónica que se despliega a favor de las idealidades y contra la densidad ontológica de la copia y del simulacro, resulta, desde mi punto de vista, especialmente certero. "¿Por qué motivo la diferencia fue subordinada a las exigencias de la representación, finita o infinita?», se pregunta en Diferencia y repetición. Deleuze responde de inmediato: «es una motivación moral en toda su pureza la que en él se declara7: la voluntad de eliminar los simulacros o fantasmas no tiene otra motivación que la moral. Lo que se condena en el simulacro es el estado de las diferencias libres oceánicas, de las distribuciones nómadas, de las anarquías coronadas, toda la malignidad que cuestiona tanto la noción de modelo como la de copia. Más adelante, el mundo de la representación podrá olvidar más o menos su origen moral, sus presupuestos morales. Estos, sin embargo, no dejarán de seguir actuando en la distinción de lo original y lo derivado, de lo original y la secuela, el fundamento y lo fundado, que anima a las jerarquías de la teología representativa, prolongando la complementariedad del modelo y la copia».

${ }^{4}$ Ibid., p. 79.

${ }^{5}$ ADORNO Th., Dialéctica negativa, Madrid, Taurus, 1975, p. 16.

${ }^{6}$ DELEUZE G., «Platón, los griegos», en Crítica y clínica, Barcelona, Anagrama, 1996, p. 192.

${ }^{7}$ En el movimiento que distingue entre copia y simulacro e incluye la referencia a la copia en el interior de una teoría de la Idea.

${ }^{8}$ DELEUZE G., Diferencia y repetición, Gijón, Júcar, 1988, p. 422. 
Acaso vaya siendo hora de clausurar el análisis del síntoma que nos ha sido útil para advertir el sentido de la tempestad que revolvió el horizonte finisecular: la misma agita y deteriora la consistencia de la Identidad, de lo Abstracto, de la Idea, y revaloriza la diferencia, la densidad ontológica de lo irreductible, de lo excepcional, de lo único. Ahora bien, para que la catástrofe advertida o sospechada no carezca de sentido es preciso que la misma se desenvuelva sobre un subsuelo agitado y fertilizante. Pues sólo en tal caso se facilitaría la pregunta sobre las posibles nuevas exigencias del pensar, sobre los retos teóricos que debiera afrontar una subjetividad de nuevo sorprendida.

La sorpresa de la subjetividad finisecular está naturalmente abonada por profundas modificaciones estructurales. Es indudable que se asistió entonces a lo que Hardt y Negri han caracterizado como «el paso del segundo paradigma (del capital) al tercero, del dominio de la industria al dominio de los servicios y la información, un proceso de posmodernización económica, o mejor aún, de informatización ${ }^{9}$. Conformación de un tercer paradigma que es el efecto de un agitarse sísmico más dilatado: intervenciones como las de E. Mandel -El capitalismo tardío-, G. Arrighi -El largo siglo XX- o L. Boltanski y E. Chiapello - El nuevo espíritu del capitalismo- dejan constancia de las nuevas perspectivas. Baste subrayar que el horizonte finisecular aparece caracterizado por una poderosa reestructuración que se ha consolidado como globalización y universalización del paradigma -que, no obstante, acaso permita hablar, como sugiere J. Estefanía, de «globalización mutilada» ${ }^{10}$-. En todo caso, es preciso reconocer que «la economía mundial está completamente trastornada, igual que lo estuvo durante la segunda mitad del siglo XIX por la segunda revolución industrial» ${ }^{11}$ por cuanto el perfil sistémico del orden tardocapitalista estaría enmarcado, a juicio de Ramonet, por las estrategias de las revoluciones tecnológica, financiera y mediática.

No obstante, lo fundamental para nosotros es detectar los efectos culturales y filosóficos que subyacen y han acompañado tal reordenación hasta el punto de socavar severamente los tradicionales planteamientos -actitud de la que acaso sea paradigmática una confesión de Rorty: "yo no deseo poner al día ni el universalismo ni el racionalismo, sino disolver a ambos y sustituirlos por una cosa distinta ${ }^{12}$. Intentaré ser sucinto, esquivando los problemas subsidiarios que plantean los dos efectos culturales y filosóficos que apuntaré a continuación.

Es preciso dejar constancia, en primer lugar, de la superior desconfianza en relación al fundamento y proyecciones de la racionalidad moderna. No se trata de una actitud inesperada. ¿Por qué aparece y se asienta la misma? La agita-

\footnotetext{
${ }^{9}$ HARDT M. - NEGRI A., Imperio, Barcelona, Paidós, 2002, p. 261.

${ }^{10}$ Cfr. ESTEFANÍA J., "La globalización mutilada», en JARAUTA F. (Ed.), Escenarios de la globalización, Caja Murcia, 1997.

${ }^{11}$ Ramonet I., "Crisis del fin de siglo», en JARAUTA F. (Ed.), Escenarios de la globalización, Caja Murcia, 1997, p. 20.

${ }^{12}$ RORTY R., Contingencia, ironía, solidaridad, Barcelona, Paidós, 1991, p. 86.
} 
ción convulsa y extremada que abona la reflexión sobre Auschwitz, convertido en referencia política y moral, deviene en ineludible presupuesto de la renovación filosófica. Los memoriales sobre Auschwitz exceden el recuerdo de la perversidad de una minoría social seducida por el himno diabólico del poder. El estremecedor testimonio de C. Lanzmann, por ejemplo, aterra: severo, dulce a veces, tenebroso con frecuencia, no pueden leerse las páginas de Shoah como una requisitoria subversiva contra el poder nacional-socialista, ya que esas sombras decrépitas o amantes del preciso olvido actúan como máquinas preparadas para el exterminio del otro, por cuanto reconocen que había razones para hacerlo. Revelación de la presencia del Mal que no es lo ajeno, lo que contagia en horas de exaltada crueldad o pereza, sino lo espontáneo del comportamiento moral. Diagnóstico sobre la espectacular invasión de una norma que no es Auschwitz, sino que ilustra la presencia en la racionalidad moderna de una excrecencia perversa. Glucksmann lo apuntó hace años: "¿cómo se puede permanecer fiel al horror que provoca la revelación de Auschwitz si no aparece esta misma repulsión ante el gulag y los encierros de los disidentes en sanatorios psiquiátricos? ¿Qué vale nuestra revuelta ante los bebés vietnamitas salpicados de napalm, si los niños ahogados en el mar de China nos dejan insensibles, si no nos dignamos manifestarnos contra el calvario afgano?» ${ }^{13}$. ¿A qué apunta esta referencia? No se trata de la recensión de un enloquecido actuar. El referente Auschwitz recomienda pensar que la racionalidad moderna deja abierto el resquicio para la legitimación de la ignominia, que quizás esté abonada por los tesoros teóricos de la modernidad, celosamente guarnecidos. Lo ha subrayado recientemente Reyes Mate al advertir que "lo que hay que entender bien es que esta tendencia monista ${ }^{14}$, negadora de la

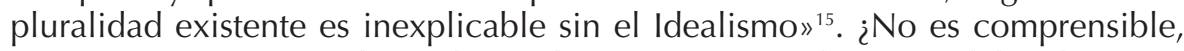
en consecuencia, que haya de producirse un grave deterioro del orden conceptual que comenzara a disponer la estrategia platónica y que mereciera una traducción efectiva en el esfuerzo cartesiano? Hardt y Negri han recordado que el nuevo horizonte de la protomodernidad "produjo una guerra ${ }^{16}$, un conflicto marcado por la ilusión reivindicativa de la inmanencia, de la singularidad, y por la inmediata contraofensiva que propusiera un poder transcendente, constituido contra un poder inmanente constitutivo, el orden contra el deseo ${ }^{17}$. ¿Es preciso señalar discursividades?: Descartes contra Montaigne, la estrategia de la nueva identidad contra la apertura de la virtù maquiaveliana.

Tal panorama se ha renovado en el tardocapitalismo. De nuevo, las exigencias de una singularidad que reivindica su densidad ontológica se manifiestan -esto es, se hacen patentes y se exponen-: el Acontecimiento 68 marcó una ple-

\footnotetext{
${ }^{13}$ GLUCKSMANN A., Contra la estupidez, Barcelona, Península, 1988, p. 213.

${ }^{14}$ La que exige la supeditación de la individualidad a la hegemonía de lo Abstracto, de la Idea -Ilámese Estado, Identidad, Raza o como se quiera.

${ }^{15}$ MATE R., Memoria de Auschwitz, Madrid, Trotta, 2003, p. 65.

${ }^{16}$ HARDT M. - NEGRI A., Imperio, p. 81.

17 Ibid., p. 81.
} 
nitud, que fuera celebración y sacramento. El segundo efecto cultural y filosófico se celebra entonces, pero no como inusitada irrupción de una palabra disidente que no se plenifica en el reconocimiento, sino en la pura y absoluta presencia. El Acontecimiento 68 fue, en efecto, evidencia de una disolución: su potencia certifica la previa conveniencia de un interés filosófico que se comprueba si se tienen en cuenta el salvamento del naufragio de las aventuras de Spinoza o Nietzsche y las significativas propuestas de Derrida o Deleuze, y, muy pronto, de Lyotard o Rorty. ¿Datos inolvidables? Derrida pronuncia su conferencia sobre la différance en enero de 1968 y Deleuze publica meses más tarde Diferencia y repetición. Lyotard y Rorty aceleran por entonces su producción teórica. El movimiento sísmico es fortísimo, y el efecto social y cultural de su virulencia es lo que denominamos posmodernidad ${ }^{18}$.

Algunos de los esfuerzos más venturosos del último tercio del XX celebraron, ciertamente, la inobjetable epifanía de la potencia de la inmanencia y el subsiguiente carácter fantasmal de lo eidético y de la representación que culmina en la radical problematización del fundamento de cualesquiera regulación o normativización. Bauman certifica cabalmente el sentido del nuevo espectáculo: «los tiempos postmodernos vienen marcados por un acuerdo prácticamente universal sobre la idea de que la diferencia no sólo es inevitable, sino que además resulta valiosa y positiva y que hay que protegerla y cultivarla» ${ }^{19}$. El propio Bauman subraya la inmediata consecuencia: «la postmodernidad... vive en un estado de apremio permanente por desmantelar toda interferencia colectiva en el destino individual, por desregular y privatizar ${ }^{20}$. No puede obviarse que tal efecto fue cuidadosamente dispuesto por la dilatada trayectoria de la aventura literaria y estética. Hassan, considerando en 1971 las producciones literarias de entonces -Beckett, Borges, Nabokov, Pinter, Cortázar, Sollers y otros muchos-, advertía en la primera edición de The Dismemberment

\footnotetext{
${ }^{18}$ Numerosos autores han subrayado la ambigüedad del concepto de postmodernidad. WELLMER A. reconocía en «La dialéctica de modernidad y posmodernidad» que «el concepto de posmodernidad o posmodernismo se ha convertido en uno de los conceptos más esquivos en la discusión estética, literaria y sociológica de la última década» -en «El debate modernidad y posmodernidad», Buenos Aires, El cielo por asalto, 1993, p. 319. Otra referencia: «si el posmodernismo cubre todo, desde el punk rock hasta la muerte de la metanarrativa, desde las revistas de historietas hasta Foucault, entonces resulta difícil ver cómo un único sistema explicativo puede llegar a hacer justicia a una entidad tan raramente heterogénea» EAGLETON T., Las ilusiones del posmodernismo, Barcelona, Paidós, 1997, p. 45. ¿Es preciso insistir? HUTCHEON L. inicia su The politics of postmodernism advirtiendo que "few words are more used and abused in discussions of contemporane culture the the world postmodernism», London, Routledge, 2002, p. 1. RIPALDA J. M. resume la problemática: «el término postmodernidad es de por sí ambiguo y utilizable como un todoterreno ideológico», De Ángelis, Madrid, Trotta, 1996, p. 58. En efecto, la variedad de los campos y los distintos enfoques parecen facilitar las observaciones de Wellmer o Eagleton: sin embargo, téngase presente que la posmodernidad, en tanto horizonte cultural y social, acoge diferentes perspectivas y, desde luego, diversas consideraciones críticas y entusiastas. Nada extraordinario. Similar situación es la que planteó a los coetáneos ilustrados la consideración de su propia actualidad.

${ }^{19}$ BAUMAN Z., La postmodernidad y sus descontentos, Madrid, Akal, 2001, p. 43.

${ }^{20}$ BAUMAN Z., La postmodernidad y sus descontentos, p. 23.
} 
of Orpheus que «indudablemente, estos nombres son demasiado heterogéneos para formar un movimiento, paradigma o escuela ${ }^{21}$. Años más tarde, en el Postface 1982, Hassan propone un concepto para facilitar la representación de la literatura postmoderna. En el añadido a la obra citada, subraya la indetermanence como referencia clave de la literatura, concepto que englobaría la indeterminacy y la inmanence ${ }^{22}$. La indetermanence recoge la expresión de las numerosas disponibilidades ontológicas y la dificultad de su formalización expresiva -ambigüedad, discontinuidad, desintegración, diferencia, destotalización, deslegitimación.

Sin embargo, serán las intervenciones filosóficas las que precisen el sentido de la indetermanence o manifiesten una notable inquietud ante las implicaciones de la disolución irrevocable del sentido. Se inaugura la aventura que pretende cartografiar el sentido de la sorpresa de las subjetividades. El desafío aceptado es fortísimo e intranquilizante: cómo operar en el mundo habitado por esa différance que "no se presenta nunca como tal. A nadie ${ }^{23}$, que no es «literalmente, ni una palabra ni un concepto ${ }^{24}$; cómo abordar la realidad de un mundo humano caracterizado por la demolición de la univocidad del ser (humano) que «se convierte en una verdadera expresión afirmativa ${ }^{25}$ que impide la representación de la diferencia expresiva; cómo facilitar la realización de los intereses del ironista liberal en tanto modelo del adecuado comportarse del ser moral cuando el mismo se afianza sobre la fuerte duda relativa a su propio léxico (Rorty). La filosofía marca el punto de inflexión. Lo que era provocación literaria o estética nutre una explosión cultural que justifica la propuesta de la tropología de H. White -el intento más provocador contra la ensoñación historicista-. O la renovación que afecta a los enfoques jurídicos y que encuentran eco en las propuestas de la Critical Legal Studies -materializadas en los trabajos de Gary Peller o Roberto Unger-, en el pragmatismo de Stanley Fish o en las inquietudes de Costas Douzinas y Peter Goodrich ${ }^{26}$.

Un nuevo panorama. Parece ineludible. Los problemas parecen acotados: pero, ¿de qué problemas puede hablarse...?

\section{Diferencia y desregulación}

Entiendo que el primer problema de la posmodernidad -esto es, de nuestra actualidad- se centra en la reflexión sobre la naturaleza de la diferencia. Una somera consideración pretende reseñar las determinaciones constituyentes. Tal

\footnotetext{
${ }^{21}$ HASSAN I., The Dismemberment of Orpheus, Univ. of Wisconssin Press, 1982, p. 260. La primera edición es de 1971.

22 Ibid., p. 269.

${ }^{23}$ DERRIDA J., "La différance», en Márgenes de la filosofía, Madrid, Cátedra, 1989, p. 41.

${ }^{24}$ DERRIDA J., «La différance», p. 42.

${ }^{25}$ DELEUZE G., Diferencia y repetición, p. 95.

${ }^{26}$ Cfr. el sucinto y certero análisis recogido en CONNOR S., Postmodernists Culture, Oxford, Blackwell, 1997, pp. 61 y ss.
} 
interés ya fuera la tarea primordial de las aventuras ontológicas de Heidegger o Sartre: el acopio de indicaciones a partir de lo que constituiría el ser-ahí o la situación resultaría interminable. La analítica de las determinaciones tiene un sentido eminentemente descriptivo, facilita el hablar sobre las apariencias y los secretos, se erige como discursividad sin término. La provocación desafiante que asume la analítica de las determinaciones puede desembocar en el libro interminable.

Es interminable porque la atención del relato resulta animada por una espectacularidad sorprendente, siempre abierta, sorpresiva por cuanto es impensable la proyección del inmediato futuro. En efecto, la posmodernidad sólo puede pensar a partir de dos presupuestos. Por un lado, del pensamiento de la muerte de Dios y, por otra parte, de la recuperación de la máxima kantiana que exige imperativamente el atrévete a pensar-actuar. Ya sabemos que la muerte de Dios "significa que el mundo suprasensible ha perdido su fuerza efectiva» ${ }^{27}$ y que la exigencia del atrévete a pensar, como sugiriera Foucault en una interesada traducción que no debemos cuestionar en este momento, invoca la urgencia de «la tarea de elaborarse a sí mismo ${ }^{28}$ que facilitaría un estar en el mundo que «is essentially a mode of self-formation ${ }^{29}$. Tales presupuestos cuestionan gravemente el panorama de las inquietudes actuales, pues la muerte de Dios y el atrévete a pensar sugieren que la reflexión sobre las determinaciones constituyentes de la diferencia no puede tener finalización. El atrévete a pensar en el horizonte de la muerte de Dios impone estrictamente la necesidad de lo interminable, pues la analítica de las determinaciones constituyentes de la diferencia, parece exigir la necesidad de lo interminable debiendo concluirse que la diferencia es, esencialmente, imprevisible y aleatoria. La imaginatio espinoziana, la nietzscheana voluntad de poder, la analítica existencial heideggeriana o la proyección del proyecto sartreano son propuestas que marcan y definen la imprevisibilidad de la subjetividad. Escribió Deleuze: «el sistema del simulacro afirma la divergencia y el descentramiento: la única unidad, la única convergencia de todas las series es un caso informal que las comprende a todas. Ninguna serie goza de ningún privilegio sobre las otras, ninguna posee la identidad de un modelo, ninguna la semejanza de una copia. Ninguna se opone a las otras, ni le es análoga. Cada una está constituida por diferencias, y comunica con las otras mediante diferencias de diferencias. Las anarquías coronadas sustituyen a las jerarquías de la representación; las distribuciones nómadas, a las distribuciones sedentarias de la representación ${ }^{30}$. Atención. Es fundamental: la densidad del simulacro potencia la divergencia y el descentramiento, esto es, la emergencia de las realizaciones del deseo y la diversidad de las potencias que producen las mismas. La diferencia es, a un tiempo, certificación analítica y proyección pensable.

\footnotetext{
${ }^{27}$ HEIDEGGER M., "La frase Dios ha muerto», en Senderos, Madrid, Alianza, 1995, p. 196.

${ }^{28}$ FOUCAULT M., «Qué es la Ilustración», en Ética, estética, hermenéutica, Barcelona, Paidós, 1999, p. 344.

${ }^{29}$ BERNAUER J. W. - MAHON M., "The Ethics of M. Foucault», en GOTTING G. (Ed.), Foucault, Cambridge U. P., 1994, p. 143.

${ }^{30}$ DELEUZE G., Diferencia y repetición, p. 439.
} 
Ahora bien, la recuperación de la inmanencia como densidad y límite ontológicos no plantea tan sólo problemas por lo que se refiere a la aventura analítica que deba desarrollarse. Las inquietudes teóricas sobresalientes se presentan en el cuestionamiento de la proyección comunitaria por cuanto el nexo causal entre Identidad y regulación de los criterios que determinan la pertinencia de sus proyecciones resulta gravemente deteriorado, siendo sustituido por la relación entre Diferencia y desregulación de los mismos. La consolidación de la inmanencia pone en cuestión cualquier pretensión fundamentadora de un horizonte comunitario y social. Es el territorio en que debe situarse la actualidad filosófica: una geografía peculiar en la que las indicaciones circulatorias han dejado de significar y en el que las rutas se despueblan. Bauman ha diagnosticado oportunamente la naturaleza del insólito horizonte: «la postmodernidad... vive en un estado de apremio permanente por desmantelar toda interferencia colectiva en el destino individual, por desregular y privatizar» ${ }^{31}$, escribe. Los campos afectados son el conjunto de los saberes que pretenden orientar la praxis humana y social. Es decir, todos. Algo al respecto se ha apuntado con anterioridad. Pero interesa en este momento introducir el radical cuestionamiento a que ha sido sometida la relación identidad-regulación. Quisiera referirme con brevedad a tres manifestaciones práxicas en las que la reivindicación de la inmanencia circunscribe la nueva problematicidad filosófica.

El primer y radical desplazamiento se plantea en la consideración de los comportamientos éticos. Conozco pocas ilustraciones tan precisas como la que dedica Bauman en Postmodern Ethics a perfilar el panorama ético de la postmodernidad. En apenas cinco páginas de la introducción a dicha obra, se ofrecen siete puntos relevantes para limitar la moral desde la perspectiva actual: viene a indicarse que el comportamiento humano es "morally ambivalent», que el fenómeno moral es «inherently non-rational», que la moralidad «ist non universalizable», que, por tanto, se problematiza fuertemente el problema del encuentro intersubjetivo y, finalmente, que el posicionamiento a favor del relativismo moral parece ser la actitud teórica mejor sustentada ${ }^{32}$. Como puede comprenderse, tal perfil teórico deriva precisamente de la analítica de las diferencias sustentada en el convencimiento del atrévete a pensar modelado por el reconocimiento de la muerte de Dios. La apropiación del todo vale como imperativo ético desarma la posibilidad de toda regulación ética. Y, si la problematicidad ética deviene inquietante, no menos perturbador resulta el panorama político. Fehér limitaba el problema al apuntar que «políticamente hablando, los que han elegido considerarse postmodernos están, en primer lugar, después de la gran narrativa» ${ }^{33}$. La gravedad del asunto no radica en el hecho advertido por el propio Fehér de que «cualquier tipo de política redentora es incompatible con la condición política postmoderna $»^{34}$, sino, más radi-

\footnotetext{
${ }^{31}$ BAUMAN Z., La postmodernidad y sus descontentos, p. 23.

${ }^{32}$ Cfr. BAUMAN Z., Postmodern Ethics, Oxford-Cambridge, Blackwell, 1993, pp. 10-15.

${ }^{3}$ FEHÉR F., «La condición política postmoderna», en Políticas de la postmodernidad, Barcelona, 1989, p. 149.

${ }^{34}$ Ibid., p. 152.
} 
calmente, que una proyección comunitaria de la diferencia resulta imposible o, más estrictamente, que su único despliegue se advierte en la traducción de lo político en términos de absoluta privacidad. La exhibición de lo privado se transforma en el esencial referente político, como ha subrayado Bauman en En busca de lo político ${ }^{35}$. De esta manera, se constata "an inevitable divorce between one's intelectual existence and one's social, ethical, and political life ${ }^{36}$. Así, y como se apuntaba en la previa y breve referencia ética, las posibilidades de una proyección política son fuertemente desactivadas como no sea que aceptemos que la única política posible es la de exhibir la existencia como obra de arte -extremando algunas observaciones nietzscheanas o ciertas sugerencias del último Foucault, perspicazmente analizado por W. Schmid en su En busca de un nuevo arte de vivir. Y no desearía omitir una somera referencia al campo estético. Es cierto que la consideración de la producción artística y literaria ha estado tradicionalmente marcada por el reconocimiento de la fundamentalidad de los procesos de subjetivización que determinan en gran medida el carácter del objeto estético. Pero, como eficazmente mostrara Bourdieu, una consideración sincrónica de las transformaciones estéticas pone de manifiesto a un tiempo la obsolescencia del gusto y la preeminencia de un gusto social en un momento dado. La apariencia postmoderna al respecto es marcadamente distinta. Bauman ha reconocido que «las nuevas creaciones artísticas no se proponen desterrar y relevar a las existentes, sino sumarse a las demás, haciéndose un hueco dentro del panorama artístico notoriamente superpoblado» ${ }^{37}$. En efecto, el panorama estético postmoderno se confirma en el eclecticismo, en la constitución de un campo estratigráfico que permite la convivencia de ofertas no sólo sumamente diferenciadas, sino también modeladas de acuerdo a criterios radicalmente enfrentados en relación a lo que sea la vivencia estética.

Tales circunstancias parecen delinear la postmodernidad como un territorio marcado por una analítica de las múltiples necesidades y de las numerosas proyecciones de lo individual. En consecuencia, se configura como máxima orientadora de las proyecciones práxicas un afianzado todo vale al que se han referido Bauman o $U$. Beck desde diferentes perspectivas e intereses. Aquel ha escrito, refiriéndose a la forma de vida regida por la incertidumbre, que «muchos dichos populares se refieren a esa clase de vida: adagios que hablan de andar a tientas, vivir para arrepentirse o tener una vida ligera; la sabiduría popular condenaba esa clase de vida, lamentaba el destino de los que la vivían y exaltaba la necesidad de evitarla ${ }^{38}$, para proceder a la crítica de la máxima reconocida. Tal recomendación de evitar el vivir a tientas ha desaparecido de nuestra actualidad. Beck, por su parte, ha remarcado la trayectoria que desemboca en el proceso de individuación que «apunta al deterioro, descomposición y desencantamiento de los magnas de sentido colectivo». La sociedad

\footnotetext{
${ }^{35}$ Cfr., por ejemplo, BAUMAN Z., En busca de lo político, México, FCE, 2001, p. 73.

${ }^{36}$ GAGGI S., Modern/Postmodern, Philadelphia, Uni. Pennsylvania Press, 1989, pp. 186-187

${ }^{37}$ BAUMAN Z., La postmodernidad y sus descontentos, p. 127.

${ }^{38}$ BAUMAN Z., En busca de lo político, p. 155.
} 
del riesgo está perfilada por el hecho de que «todos los esfuerzos de definición se concentran en el individuo» ${ }^{39}$.

Es indudable que el horizonte postmoderno aparece limitado por la revalorización de la inmanencia y el replanteamiento de la posibilidad de una articulación colectiva o la apuesta a favor de la aceptación de la plena soberanía de lo privado, cuyo manifestarse explotaría las posibilidades exclusivas de la política. "Cualquier tipo de política redentora es incompatible con la condición política postmoderna», advertía F. Fehér con pesar. Podría extremarse el diagnóstico: cualquier tipo de proyección comunitaria es incompatible con la condición postmoderna. Sin embargo, debe acentuarse la prudencia a la hora de dibujar el panorama postmoderno. Pues entiendo que la revalorización de la inmanencia y la consecuente aceptación de los procesos de desregulación ética, política, estética- subraya precisamente el problema esencial de la postmodernidad. ¿De qué se trata? Desde mi punto de vista, el problema esencial es el de pensar el efecto de la desregulación a partir de la consideración de la aceptación del valor ontológico de la inmanencia. Y, en tal sentido, numerosas críticas de la actitud postmoderna carecen de cautela: Eagleton, Jameson, Habermas, Fehér, Norris, Bauman o Gaggi -por citar algunos estudiosos que nos han acompañado en estas páginas- presuponen que la reflexión en la postmodernidad desemboca necesariamente en el todo vale. Nada más inexacto, pues es preciso advertir que el panorama filosófico de la postmodernidad está animado por una controversia radical. Resulta improcedente sugerir, como hace, por ejemplo, Jameson en Las semillas del tiempo que estemos situados en la problematicidad antinómica caracterizada por la exigencia derivada de la proyección ontológica de la diferencia y la constatable estandarización que reniega de la diferencia misma. Siendo incontestable la descripción, no puede aceptarse la caracterización de la misma en tanto antinómica. Jameson se equivoca, pues lo que revela el conflicto diferencia-estandarización es, estrictamente, el conflicto interno y propio de la reflexión postmoderna en lo que hace referencia a la urgencia de pensar y proyectar modelos ético-políticos, y estéticos, que, asentados en una filosofía de la inmanencia, no desemboquen en la valorización del andar a tientas. No se trata de una antinomia postmoderna, sino en verdad de opciones ideológicas. Por esto mismo, puede advertirse en el giro postmoderno un marcado interés por articular diferencia y proyección colectiva. Y tal interés es tan constitutivo de la reflexión postmoderna como lo es el elogio del todo vale que inquieta a Bauman, Eagleton o al propio Jameson. ¿O acaso puede desconocerse que la propuesta del ironismo liberal de Rorty, las sugerencias de Deleuze o la reivindicación del telos materialista que debiera animar la constitución comunitaria en el proyecto de Negri, apuntan precisamente a la articulación de la ontología constitutiva y la proyección de un ser social? ¿Acaso puede obviarse que la reflexión postmoderna, cuando recupera a Lévinas o Blanchot, e, ilusionada, homenajea a Spinoza, está pendiente de la necesidad de afrontar la impertinencia del todo vale? Considero

\footnotetext{
${ }^{39}$ BECK U., «Teoría de la sociedad del riesgo», en BERIAIN J., (Ed.), Las consecuencias perversas de la modernidad, Barcelona, Anthropos, 1996, pp. 204-205.
} 
que este interés marca la geografía del mapa cultural postmoderno. Se trata de una línea, una marca en tal panorama que circula junto a la aceptación de la desregulación y el todo vale. Eagleton escribía: «se puede aventurar, en una primera y cruda aproximación, que gran parte del posmodernismo es políticamente opositor pero económicamente cómplice. Sin embargo, esto requiere una mayor afinación. El posmodernismo es radical en tanto desafía a un sistema que todavía necesita de valores absolutos, de fundamentos metafísicos y de sujetos autoidénticos; contra eso lanza la multiplicidad, la no identidad y la transgresión, el antifundamentalismo, el relativismo cultural. El resultado, en su mejor parte, es una subversión llena de recursos contra el sistema dominante de valores, al menos en el nivel de la teoría ${ }^{40}$. Pero es preciso aventurarse más allá. Cedo la palabra a Negri, quien ha advertido que la situación posmoderna «encamina a las subjetividades y al pensamiento crítico hacia una nueva tarea: la construcción de aquéllas como nuevas máquinas de una producción positiva del ser que no puede expresarse sino como una nueva constitución, como una revolución radical $»^{41}$. La postmodernidad reinterpreta los conflictos sociales, la conveniencia de la realización de la vida y las condiciones de una socialización que la permita. Desearía referir algunas propuestas ${ }^{42}$.

\section{Retos en el horizonte postmoderno}

Es necesario retornar a la consideración que determina la desregulación y que provoca esa inquietud que anima el replanteamiento de los procesos de socialización. Me refiero a lo que puede catalogarse como analítica de la diferencia, intervención que se convirtió en una de las operaciones centrales de la actividad filosófica a lo largo de la segunda mitad del siglo pasado. Como apuntaba Deleuze, se trataba del aire de la época que se hacía presente en numerosos signos: entre otros, en «la orientación de Heidegger hacia una filosofía de la diferencia ontológica $»^{43}$. Es indudable: la concepción heideggeriana del mundo en tanto representación de mi mundo, que delinea las fronteras de la analítica existencial, abre el camino a la exigencia de (e-)numerar los elementos constituyentes de la singularidad humana. Prolífico desafío que, por ejemplo, ahonda Sartre al remarcar la situación como el origen psicológicopráxico del existente irreductible. Y ni que decir tiene que el proceso cultural acelerado por la desintegración crítica de la estabilidad social y política que se acentúa a partir de los años 60 abundaría en la cuestión. No se trata de una marca exclusiva del perfil de la actualidad filosófica. La filosofía de la inma-

\footnotetext{
${ }^{40}$ EAGLETON T., Las ilusiones del posmodernismo, Barcelona, Paidós, 1997, p. 195.

${ }^{41}$ NEGRI A., El trabajo de Dionisos, Madrid, Akal, 2003, p. 19.

${ }^{42}$ He abordado la cuestión en otros escritos. Cfr. "Ontología de la diferencia y legitimación moral», en Praxis, Lisboa, Centro de Filosofía de la Universidade de Lisboa, 2000; "Contra el caos (a la sombra de G. Deleuze)», en Microfisuras, $\mathrm{n}^{\circ} 7$; en «Retos de la ontología constitutiva», en Riff-Raff, $\mathrm{n}^{\circ}$ 15. Igualmente y de forma más detenida en los últimos capítulos de Mirada, escritura, poder. Una relectura del saber occidental, Barcelona, Bellaterra, 2002.

${ }^{43}$ DELEUZE G., Diferencia y repetición, p. 31.
} 
nencia -sustentada en la noción epicúreo-lucreaciana del azar, en la exhibición del yo en Montaigne, desarrollada en la Ética spinoziana o en los textos del materialismo francés del XVIII- siempre discurrió paralela a la filosofía de la identidad, si bien su destino fuera el de la sombra y el ocultamiento.

Pues bien, entiendo que la analítica de la diferencia debe ahondar la operación descriptora de las determinaciones que configuran las subjetividades, pues dicha tarea es imprescindible para plantearse el asunto fundamental de la actividad filosófica, que no es otro que el de sopesar las posibilidades de la socialización. Y es en la atención a esta cuestión donde los conflictos se radicalizan. Respecto a las preguntas que ahora planteamos -cómo opera la diferencia, qué sentido tiene el planteamiento de su socialización...-, entiendo que la actualidad filosófica ha diseñado tres ofertas.

Una primera respuesta ha abordado la cuestión diferenciando con cuidado el hecho de la realización de la diferencia y el hecho de la relación entre las diferencias. Entiendo que han sido Foucault y Deleuze quienes subrayaron con cuidado hasta qué extremo la tensión de la inmanencia busca su realización. Es preciso reconocer que la diferencia constituida, activada como expresión del ser que agota en sí misma la Idea platónica, o, si se quiere, que glorifica el simulacro ${ }^{44}$, determina la exigencia plenaria de la realización individual. Tal convencimiento es el que marca la orientación del tardío Foucault ético: "cuando Foucault habla de ética, hay que comprenderla en los términos de una ética individual, de una ética entendida como arte de vivir ${ }^{45}$. Foucault habría aceptado tal valoración y, de hecho, en una entrevista concedida en abril de 1983, admitía que «nous devons faire de nous-mêmes une œuvre d’art» ${ }^{46}$. Ahora bien, ¿qué línea marca el hecho de la realización de la diferencia en relación a la realización de la otra diferencia...? Situados en el panorama que ahora discernimos brevemente, es preciso concluir que en pura lógica la relación entre las diferencias marca una figura de pronunciadas y aceleradas divergencias: la realización de la inmanencia que es revelación y constitución de sí misma sólo puede marcar la orientación del alejamiento y la extrañeza. Lo remarca con severidad Deleuze en un fragmento previamente trascrito en el que, refiriéndose al sistema del simulacro, subrayaba el movimiento específico de divergencia y descentramiento que el mismo ejecuta. Esto es, alejamiento, autoconstitución de espacios alejados que se confirman, estrictamente, en la exhibición de la aventura de las subjetividades. Toda afirmación de la inmanencia acumula extrañezas, inaugura y profundiza la verdad de la inmanencia que requiere situarse como lo que la misma expresa, es decir, evidencia de una autoconstitución incomparable. Y, sin embargo, es innegable que Foucault y Deleuze abogan por la socialización de un modelo comunitario que estaría conceptualizado, por ejemplo, en el agenciamiento del último: «un agenciamiento es una multitud que comporta muchos términos heterogéneos, y que

\footnotetext{
${ }^{44}$ Cfr. DELEUZE G., Diferencia y repetición, p. 132.

${ }^{45}$ SCHMID A.., En busca de un nuevo arte de vivir, Valencia, Pre-textos, 2002, p. 33.

${ }^{46}$ FOUCAULT M., «À propos de la généalogie de l'éthique: un aperçu du travail en cours», en Dits et écrits. IV, Paris, Gallimard, 1994, p. 392.
} 
establece uniones, relaciones entre ellos, a través de edades, de sexos y de reinos -a través de diferentes naturalezas. La única unidad del agenciamiento es de co-funcionamiento: una simbiosis, una simpatía. Lo importante no son las filiaciones, sino las alianzas y las aleaciones; ni tampoco las herencias o las descendencias, sino los contagios, las epidemias, el viento ${ }^{47}$. ¿Cómo se realiza el mismo? Acaso sólo en la aleatoriedad y la espera. Quizás sólo, como ilustrara Blanchot, tan admirado por uno y otro, en un encuentro inesperado como el vivido en el 68- cuando «le Dire primait le Dit» ${ }^{48}$.

De tal manera que parecen oportunas las observaciones de Hardt y Negri referidas a Foucault, Deleuze y Guattari, cuando observan que, culminando un pertinente análisis del horizonte biopolítico, «terminan articulándolo sólo de un modo superficial y efímero, como un horizonte caótico, indeterminado, caracterizado por un acontecimiento inasible ${ }^{49}$. Es necesario cuestionar la aleatoriedad del encuentro entre las diferencias. Así, se dibuja una segunda alternativa que, desde mi punto de vista, se asienta en la reconsideración de la pertinencia de una Totalidad-Totalización que legitimara y fundamentara un proyecto comunitario. Es la aventura central que ha abordado Negri en la última década. Situándose en el reconocimiento de la realidad económico-política de lo que se configura como Imperio, pueden detectarse movimientos de resistencia que podrían dar forma a una nueva multitudo, asumiéndose que «ser republicano hoy significa ante todo luchar dentro del imperio y construir en su contra, sobre sus terrenos híbridos y cambiantes. Y aquí deberíamos congregar, contra todos los moralismos y todas las posiciones de resentimiento y nostalgia, que este nuevo terreno imperial ofrece mayores posibilidades de creación y liberación. La multitud, su voluntad de estar en contra y su deseo de liberación deben atravesar con esfuerzo el imperio para salir del otro lado $»^{50}$. Observación suscrita con anterioridad. Negri ya se había referido en Fin de siglo a la presencia de un sujeto nuevo «que nace de las cenizas del antiguo, pero que, precisamente por ello, vuelve a determinar la posibilidad como horizonte allí donde el antiguo sujeto había concluido en la necesidad y su apreciación había terminado en el encantamiento ${ }^{51}$. Omitiré toda referencia a la relación entre este nuevo sujeto constituyente de la renovada multitudo y a la relación entre el mismo y las exigencias condicionadas por el esfuerzo a favor de satisfacer las necesidades obstruidas por las condiciones reales, para centrarme en algunos puntos de su reflexión en torno a la posibilidad y fundamentación de la proyección comunitaria -que, como se ha apuntado, se presenta con un esencial grado de aleatoriedad en Foucault y Deleuze-. Pues bien, es en un breve y poco conocido opúsculo, publicado poco antes de Imperio, donde Negri presenta una panorámica de notable claridad. «La questione era

\footnotetext{
${ }^{47}$ DELEUZE G. - PARNET C., Diálogos, Valencia, Pre-textos, 1980, p. 79.

${ }^{48}$ BLANCHOT M., La communauté inavouable, Paris, Minuit, 1983, p. 52.

${ }^{49}$ HARDT M. - NEGRI A., Imperio, p. 42.

50 Ibid., p. 206.

${ }^{51}$ NEGRI A., Fin de siglo, Barcelona, Paidós, 1989, p. 36.
} 
semplice e dificilisima da risolvere: como si fa una decisione della moltitudine? $\aleph^{52}$, se pregunta. Las páginas de Kairòs, alma Venus, multitudo resumen viejas preocupaciones negrianas y, a un tiempo, esclarecen cuestiones con anterioridad no elucidadas. Para nosotros, y esencialmente, las referidas al mecanismo constituyente de una renovada multitudo. Pues bien, en el fragmento 9 sex acepta Negri que «se nella modernità la moltidudine (la masse) era prodotta dall'esterno, nella postmodernità la moltitudine si forma spontaneamente $»^{53}, y$, páginas más adelante, que «il potere constituente è la dimensione politica aderente allo sviluppo della teleologia del comune ${ }^{54}$, instaurándose de esta manera un horizonte desplegado cuya realización garantiza la potencia constituyente de la multitudo y que es legitimada por la necesidad del Amor que no es ni pietas ni amore, sino "lavoro vivo biopolitico» ${ }^{55}$. Así, las inconveniencias señaladas a propósito de Foucault y Deleuze parecen esquivarse al asentarse el proyecto comunitario en la conjunción determinada por el Amor que, animado por los puntos de encuentro que se producen en el trabajo vivo, revela una teleología de lo común que ya no es pura aleatoriedad.

La alternativa de Negri -en la trayectoria de Foucault y Deleuze- parece superar la aleatoriedad deleuziana. Sin embargo, otros problemas se plantean, pues la referencia al telos materialista introduce una renovación de la clausurada idea de la Totalidad-Totalización amparada por la articulación de la espontaneidad ${ }^{56}$ y del Amor como producción de la aproximación de las subjetividades nómadas. Dicha opción, que se alinea en el sentido de las propuestas de Anderson, Eagleton o Jameson -pese a sus marcadas diferencias: hablo de un horizonte...-, facilita las alternativas de algunas versiones del neoliberalismo y del neopragmatismo. Las manifestaciones más elaboradas son las de Rawls y Rorty. Éste último, con marcado interés y subrayando la absoluta contingencia de creencias y programas, que se extrema en la presentación del perfil del ironista radical ${ }^{57}$, sostiene que el encuentro intersubjetivo pudiera sustentarse en la aceptación de algunos valores propuestos por los poetas vigorosos y los revolucionarios ${ }^{58}$. Se trataría de atender a las exigencias de la realidad social y operar en consecuencia para articular los procedimientos tendentes a la realización de la utopía que vertebrara la defensa de la autonomía subjetiva. Y entiendo que el liberalismo de Rawls atiende a similares exigencias. En efecto, la propuesta del consenso entrecruzado parte del reconocimiento de la sociedad plural y de la propuesta de configuración de un aparato que conjugara las diferencias razonables caracterizadas por su común situarse

\footnotetext{
${ }^{52}$ NEGRI A., Kairòs, alma Venus, multitudo, Roma, Manifestilibri, 2000, p 12.

53 Ibid., p. 133.

54 Ibid., p. 136.

${ }^{55}$ Ibid., p. 146.

${ }^{56}$ Ibid., p. 133.

${ }^{57}$ RORTY R., Contingencia..., p. 91.

${ }^{58}$ Ibid., p. 79
} 
en la tolerancia ilustrada ${ }^{59}$. Así, reconocimiento de las diferencias y modelo pragmático-liberal fuerzan el perfil de un horizonte que fundaría la intersubjetividad política.

Las tres referencias incluidas piensan la necesidad de la proyección socializada de las diferencias. Y, sin embargo, los fallos de sus modelos respectivos son radicales o presentan notables insuficiencias. Enumeración somera y precipitada: las cautelas recomendadas por Rawls para determinar la razonabilidad de las diferencias y garantizar la operatividad del consenso entrecruzado eluden la caracterización esencial de las diferencias mismas y, en semejante medida, la propuesta de Rorty tan sólo puede operar a partir del reconocimiento de la superioridad de los valores articulados a partir de la tradición occidental. «Nosotros, epígonos de Platón», invoca Rorty sin precaución algu$n^{60}$. No resultan inconvenientes, en consecuencia, las reiteradas críticas de Chantal Mouffe, centradas de forma especial en subrayar el ocultamiento a que es sometida la política en uno y otro. Advertencia que, por supuesto, no podría extenderse a las propuestas que reivindican una nueva Totalidad-Totalización, como me parece ser la de Negri. Pero la improcedencia de las implicaciones políticas, de Kairòs a Imperio, puede advertirse si se cuestiona la fortaleza teórico-política del telos materialista que garantiza la proyección de la multitudo. Nada permite advertir la potencia del Amor -negriano-, cuya fortaleza sobrevalora una necesidad finalista en las subjetividades. La expresión de buenas intenciones sólo está avalada por la elucubración relativa a la presencia de un telos y por la devaluación de la relación entre la lógica económico-política del Imperio y la proyección de las subjetividades, que se supone tan esencialmente conflictiva que se alimenta el mesianismo que achacaba Danilo Zolo a la propuesta de Negri en la entrevista publicada en Da Reset. De nuevo, aunque desde otra perspectiva -y aunque pudiera parecer sorprendente-, la analítica negriana de la diferencia evita la consideración de las conflictividades que han de surgir en el despliegue mismo de las reales diferencias subversivas.

Entiendo que la aventura deleuziana es, al respecto, la más consecuente, aunque insuficiente: reconoce las diferencias, sugiere el carácter esencial de su proyección divergente, recomienda la aproximación entre las líneas de fuga. Suscribe la radical aleatoriedad de una aproximación que garantizaría la dimensión inacabable y sólo imaginable de la producción de deseo. Deleuze sitúa la problemática para postmodernidad en otro ámbito, ya que es quien plantea con más consistencia, advertido en el subsuelo y en la reivindicación de su rincón, la dificultad de la aproximación de la plural producción de deseo. No toda diferencia es federable. Precisa la espera, la admiración, la consistencia del retraso. No hay apuesta posible a favor de una razonabilidad, no hay telos materialista. El problema de la insuficiencia teórico-política deleu-

\footnotetext{
${ }^{59}$ Cfr. HABERMAS J. - RAWLS J., Debate sobre el liberalismo político, Barcelona, Paidós, 1988, p. 55 ${ }^{60}$ RORTY R., Consecuencias del pragmatismo, Madrid, Tecnos, 1996, p. 257.
} 
ziana radica en el hecho de que renuncia a la intervención referida a las condiciones de posibilidad del agenciamiento que construyen las plurales líneas de fuga contra la fijación paranoico-fascista, despótica ${ }^{61}$.

Los retos filosóficos del horizonte postmoderno están limitados por las dos inquietudes que hemos diseñado: (e-)numeración de las diferencias y apuesta por una articulación socializada. La satisfacción que produce la consideración del primer aspecto es de la misma dimensión que la intranquilidad que siembra la atención a la segunda. Es incuestionable que la analítica de la diferencia no puede desembocar en la mera (e-)numeración de los efectos de la apertura ontológica, pero las propuestas de Negri, Rawls o Rorty reniegan, precisamente, del ser mismo de la diferencia al tachar lo político o al reconocer un telos que determinaría la aproximación entre los adversarios de la lógica del Imperio. Por su parte, la reflexión deleuziana desemboca en una espera tan confiada cuanto amarga al estar asentada en la sospecha de que la aleatoriedad se ha de resolver en orden al favor positivo referente a la producción de deseo y, a un tiempo, en la aceptación de que nada puede afirmarse ni debe hacerse a favor de la aceleración de la socialización contra la fijación paranoico-fascista.

Pues bien, entiendo que la problemática política postmoderna debe afrontar la evaluación de las diferencias, ciertamente, para, por cuanto toda diferencia se presenta como aventura de divergencia y descentramiento en relación a la otra diferencia, proceder a la reflexión relativa a la conveniencia de recomendar la corrección de la divergencia-descentramiento. Las posibilidades sólo pueden ser dos: confiar en la contaminación -en el viento, ha metaforizado Deleuze- o potenciar la convergencia. ¿Por qué no apostar por una conveniencia estratégica? A nadie compete el diseño de la misma. Sin embargo, pueden marcarse las cautelas que una tal conveniencia estratégica debiera respetar. ¿Alguien puede hacerlo? ¿O limitarse a sugerir determinadas exigencias?

Quisiera subrayar cuatro mínimas exigencias. En primer lugar, es preciso renovar el interés primario de la analítica de las diferencias, que no debe ser restringida a la función (e-)numerativa: una analítica de las diferencias materialista debe certificar la existencia de las diferencias y, a un tiempo, evaluarlas a partir de la relación entre las diferencias mismas y de la relación entre las diferencias y la diferencia dominante constituida como Identidad. Por otra parte, es urgente proceder a la catalogación de las diferencias (e-)numeradas que sería el resultado de la previa evaluación: tal operación permitiría reconocer dos tipos de relación, una de las cuales marcaría la identidad del enemigo y otra marcaría el perfil del adversario ${ }^{62}$. Tercera exigencia: la aproximación al

\footnotetext{
${ }^{61}$ Deleuze y Guattari entendían por «fijación paranoico-fascista» la determinación que rige los comportamientos ofensivos contra toda manifestación de la diferencia en virtud de su asentamiento en los cánones producidos para orientar la praxis y controlar la producción de deseo. Respeto a lo largo del texto la caracterización, que se abre paso en El Antiedipo a pesar del posible confusionismo que la misma produce.

${ }^{62}$ Recojo esta diferenciación de MOUFFE Ch. cfr. especialmente La paradoja democrática, Barcelona, Gedisa, 2003, pp. 114-115
} 
adversario, que sustenta el proyecto político de Ch. Mouffe, o la espera para situarse ante la federación de diferencias (Deleuze), plantea acaso el conflicto más grave de una proposición estratégica contra la fijación paranoico-fascista, puesto que, respetando el sentido de la diferencia, se constata que toda diferencia diverge, afianza la producción de deseo como necesidad autoconstitutiva. La radicalidad del problema es transparente. Una aporía esencial parece asentarse: es preciso realizar la producción de deseo -esto es, reconocer la divergencia- $y$, a un tiempo, oponer la pertinencia estratégica de la aproximación. Como es sabido, un conflicto aporético tan sólo se resuelve optando a favor de uno de los elementos que constituyen el mismo. La apuesta que debe afrontarse entonces es la siguiente: ¿la producción de deseo desembocará en la aproximación o, diversamente, la pertinencia estratégica de la aproximación facilitará la producción de deseo? Se trata de una apuesta, y todo parece indicar que el segundo término de la antinomia resolverá la producción de deseo, mientras que la producción de deseo no determina la aproximación. Por esto mismo, entiendo que es urgente abundar en dicha orientación. Lo que presupone un cierto grado de aminoración o de puesta en suspenso de los aspectos que dificultarían la federación de las diferencias. Dicha recomendación debe tener un sentido. Pues bien, es el análisis del horizonte social y político de la postmodernidad, la realidad del Imperio, lo que aconseja la pertinencia de tal puesta en suspenso de lo que acentúa la divergencia y el descentramiento. Chantal Mouffe ha denominado tal horizonte como pluralismo agonístico. Por mi parte, he insistido en otros textos sobre el carácter de coyuntura trágica que significa necesariamente la aceptación por parte de las subjetividades de la necesidad de una coyuntural detención de sus proyecciones que podría ser factible en virtud de la asunción de la oposición a la estrategia neoidentitaria como parte constitutiva de toda diferencia federable.

¿Resolvería dicha estrategia las dificultades que el problema de la socialización de las diferencias plantea a la actualidad filosófica postmoderna? Se trata de una propuesta estratégica y, en tanto tal, no supera el ámbito de un ensayo inspirado en la necesidad de encontrar una posibilidad política que favorezca la presencia y aceptación social de las diferencias. ¿Resolvería dicha estrategia las dificultades que el problema de la socialización de las diferencias plantea a la actualidad filosófica postmoderna?, repito. Ah, el viento... Pero, ahora, un viento que podría comenzar a soplar sobre nuestras cabezas. 